\title{
COEFICIENTE DE DETERMINAÇÃO GENOTÍPICA E DE DIVERSIDADE GENÉTICA EM TOPOCRUZAMENTOS DE SOJA TIPO ALIMENTO COM TIPO GRÃO1
}

\author{
GILBERTO KEN-ITI YOKOMIZO² e NATAL ANTONIO VELLO 3
}

\begin{abstract}
RESUMO - As estimativas do coeficiente de herdabilidade são importantes para a escolha de uma estratégia eficaz de seleção. Quando os tratamentos são considerados fixos, a herdabilidade é denominada coeficiente de determinação genotípica. Os objetivos do presente trabalho foram estimar, através de topocruzamentos obtidos entre soja tipo alimento e soja tipo grão, o coeficiente de determinação genotípica e avaliar a diversidade genética de modo a se distinguir diferenças de contribuição dos parentais de soja tipo alimento com sementes grandes e com sementes pequenas usados nos topocruzamentos, assim como avaliar a interação de cada grupo com o ambiente. Os resultados de determinação genotípica obtidos nas médias de topocruzamentos foram: $90,51 \%$ com relação ao número de dias para maturidade (NDM); 93,92\% com relação à altura da planta na maturidade (APM); $79,52 \%$ com relação ao acamamento (AC); $91,37 \%$ ao valor agronômico (VA); $95,55 \%$ ao peso de cem sementes (PCS); 57,57\% à produtividade de grãos (PG), e 91,18\% à largura visual das vagens (LVV), enquadrando-se aos valores encontrados na literatura. Em relação às estimativas de diversidade genética, que representam a disponibilidade de diferenças genéticas para seleção, os caracteres NDM, APM e AC apresentaram valores maiores nos topocruzamentos envolvendo parentais exóticos de sementes pequenas e para VA, PCS, PG e LVV nos topocruzamentos envolvendo parentais exóticos de sementes grandes.
\end{abstract}

Termos para indexação: Glycine max, progênie, seleção, variação genética, métodos de melhoramento.

\section{GENOTYPIC DETERMINATION COEFFICIENT AND GENETIC DIVERSITY AT TOPCROSSES BETWEEN FOOD TYPE AND GRAIN TYPE SOYBEAN}

\begin{abstract}
The estimates of the heritability coefficient are necessary for the choice of an efficient strategy of selection. When the treatments are considered fixed, the heritability is called genotypic determination coefficient. In the present work the genotypic determination coefficient and the genetic diversity to distinguish the differences between genetic contribution of exotic parentals from type food soybean with great seeds and small seeds in topcrosses with two adopted type grain parentals and the interactions with the environment were studied. The results of genotypic determination for the topcross averages was $90.51 \%$ for number of days for maturity (NDM); $93.92 \%$ for height of the plant at maturity (APM); $79.52 \%$ for lodging (AC); $91.37 \%$ for agronomic value (VA); $95.55 \%$ for one hundred seed weight (PCS); $57.57 \%$ for seed yield (PG) and $91.18 \%$ for pod width by visual score (LVV), that agree with the values found in literature. In relation to the estimates of genetic diversity, that represent the availability of genetic differences for selection, the characters NDM, APM and AC, showed higher values in the small seed of exotic parental topcrosses and for VA, PCS, PG and LVV in the great seeds of exotic parental topcrosses.
\end{abstract}

Index terms: Glycine max, progeny, selection, genetic variation, breeding methods.

\footnotetext{
${ }^{1}$ Aceito para publicação em 18 de fevereiro de 2000.

${ }^{2}$ Eng. Agrôn., Dr., Embrapa-Centro de Pesquisa Agroflorestal do Amapá (CPAFAP), Caixa Postal 10, CEP 68902-280 Macapá, AP. E-mail: gilberto@ cpafap.embrapa.br

${ }^{3}$ Eng. Agrôn., Prof. Titular, Dep. de Genética, Escola Superior de Agricultura Luiz de Queiroz (ESALQ), Caixa Postal 83, CEP 13400-970, Piracicaba, SP. E-mail: naavello@carpa.ciagri.usp.br
}

\section{INTRODUÇÃO}

A soja (Glycine $\max$ (L.) Merrill) constitui uma fonte alimentar protéica de grande importância mundial, representando atualmente a maior porcentagem de concentrados protéicos destinados para a alimentação animal e humana (Carrão-Panizzi, 1988). 
Por este motivo, grandes esforços e investimentos são empregados no melhoramento genético da cultura.

Para que ocorra um processo seletivo eficiente das populações segregantes de soja, deve-se acompanhar e compreender as alterações de comportamento, devido às diferentes características intrínsecas a cada conjunto de genótipos. Assume grande importância a variabilidade genética disponível e as estimativas de herdabilidade dos caracteres de interesse. A estimação do coeficiente de herdabilidade, referente aos caracteres em estudo, é necessária para a escolha adequada de uma estratégia eficaz de melhoramento genético, permitindo a seleção, precoce ou não, de materiais, e a intensidade de seleção a ser aplicada.

O melhoramento de caracteres como produtividade de grão, altura da planta na maturidade, acamamento da planta na maturidade, ciclo da planta e tamanho das sementes são fundamentais para a obtenção de novas variedades competitivas em relação às já existentes. Sabe-se que em caracteres com herdabilidade baixa, a eficiência do processo de seleção é dificultada, principalmente em face da influência ambiental, que ao interagir com os genótipos pode alterar as estimativas de herdabilidade. Johnson et al. (1955) citam que com uma maior diversidade de ambientes em estudo, aumenta-se a precisão da estimativa de herdabilidade dos caracteres avaliados, possibilitando melhor compreensão deles.

A soja pode ser didaticamente classificada em dois grupos principais: tipo grão e tipo alimento (Vello, 1992). A soja tipo grão tem sido cultivada, no Brasil, principalmente para atender às indústrias brasileira e estrangeira de farelo e óleo; suas características são: tamanho médio das sementes, representado pelo peso de cem sementes (PCS) entre 10 e $19 \mathrm{~g}$, e gosto desagradável ao consumo humano direto. Já a soja tipo alimento, que apresenta sabor agradável ao consumo humano direto, é dividida em duas categorias: a) sementes pequenas com PCS menor que $10 \mathrm{~g}$, destinadas ao consumo em forma de brotos e de "natto" (fermentado); b) sementes grandes com PCS igual ou maior que $20 \mathrm{~g}$. A soja alimento, tem os seguintes usos principais: $\mathrm{b}_{1}$ ) soja tipo hortaliça (vegetable soybean, green soybean, ou edamame), com consumo de grãos obtidos de vagens imaturas, estádio $\mathrm{R}_{6}$ de Fehr \& Caviness (1977); $b_{2}$ ) soja tipo doce (kuromame), com grãos maduros de tegumento preto, usados na preparação de doces especiais; $b_{3}$ ) soja tipo salada, com grãos maduros, de tegumento e hilo de coloração clara (preferencialmente creme ou amarela, em algumas regiões também se usam grãos de cor verde ou variegada), para preparação de saladas mistas, junto com hortaliças e legumes.

Os objetivos deste trabalho foram estimar, através de topocruzamentos obtidos entre soja tipo alimento com soja tipo grão, o coeficiente de determinação genotípica e a diversidade genética de modo a se distinguir diferenças de contribuição dos parentais de soja tipo alimento com sementes grandes e com sementes pequenas usados nos topocruzamentos, assim como avaliar a interação de cada grupo com o ambiente.

\section{MATERIAL E MÉTODOS}

$\mathrm{O}$ experimento foi conduzido em área pertencente ao Departamento de Genética da ESALQ/USP em Piracicaba, SP. O material genético compreendeu 72 topocruzamentos em geração $\mathrm{F}_{5: 4}: 67$ provenientes do cruzamento de materiais exóticos tipo hortaliça, de sementes grandes, sendo obtidos 30 com a cultivar FT-2, e 37 com a cultivar Doko; e cinco com materiais exóticos de sementes pequenas do tipo broto/"natto", sendo três com Doko e dois com FT-2.

A semeadura foi realizada no verão, em três datas (30 de outubro, 5 e 20 de novembro), constituindo o efeito de ambientes, e cada planta $\mathrm{F}_{4}$ foi representada por uma cova com uma a cinco plantas $F_{5}$. A parcela foi composta por três fileiras com quatro covas cada, perfazendo 12 covas por repetição de cada topocruzamento. $\mathrm{O}$ espaçamento utilizado foi de $0,5 \mathrm{~m}$ entre covas, e $0,5 \mathrm{~m}$ entre fileiras. O delineamento adotado foi o de blocos ao acaso, com duas repetições por época, totalizando 72 covas por topocruzamento.

Os tratamentos $\left(\mathrm{G}_{\mathrm{i}}\right)$ foram considerados como fixos, e avaliados no ano agrícola 1991/92, estimando-se os coeficientes de determinação genotípica $\left(\mathrm{R}^{2}\right)$ e de diversidade genética.

O modelo linear estatístico adotado foi o proposto por Vencovsky (1992):

$\bar{Y}_{i j k}=m+G_{i}+E_{j}+(G E)_{i j}+B_{k(j)}+\bar{E}_{i j k}$ sendo:

$\overline{\mathrm{Y}}_{\mathrm{ijk}}=$ valor fenotípico médio do caráter $\mathrm{Y}$ medido no i-ésimo genótipo, no j-ésimo ambiente; 
$\mathrm{m}$ : média geral de todos os i-ésimos genótipos avaliados; $\mathrm{G}_{\mathrm{i}}$ : efeito fixo do i-ésimo genótipo, $(\mathrm{i}=1,2, \ldots, \mathrm{g})$; $E_{j}$ : efeito aleatório do j-ésimo ambiente, $(j=1,2, \ldots, e)$; $(\mathrm{GE})_{\mathrm{ij}}$ : efeito aleatório da interação do i-ésimo genótipo com o j-ésimo ambiente;

$\mathrm{B}_{\mathrm{k}(\mathrm{j})}$ : efeito aleatório do k-ésimo bloco dentro do j-ésimo ambiente, $(\mathrm{k}=1,2, \ldots, \mathrm{r})$;

$\overline{\mathrm{E}}_{\mathrm{ijk}}$ : erro aleatório médio associado à observação.

Os caracteres avaliados foram: número de dias com relação à maturidade (NDM), contados desde a semeadura até o momento de colheita; valor agronômico (VA), avaliado na maturidade por escala de notas visuais de 1 (planta ruim) até 5 (planta ótima), com base em características agronômicas da planta, como arquitetura, sanidade, produtividade, presença ou não de retenção foliar e haste verde; produção de grãos (PG), avaliado pelo peso dos grãos, em gramas, de cada planta; peso de cem sementes (PCS), avaliado pela pesagem de uma amostra aleatória de cem sementes para cada cova, em gramas, e largura visual das vagens (LVV), avaliado na maturidade por uma escala de notas visuais de 1 (vagem estreita) até 5 (vagem larga).

A análise de variância simplificada, desdobrando-se os tratamentos com suas respectivas interações, foram obtidos segundo a Tabela 1.

Os valores dos coeficientes de determinação genotípica foram estimados a partir da análise de variância para cada caráter, conforme procedimento das estimativas de diversidade genética apresentado na Tabela 2.

TABELA 1. Obtenção das estimativas de diversidade genética e variâncias com os desdobramentos de tratamentos em grupo dos topocruzamentos de sementes grandes (g), grupo dos topocruzamentos de sementes pequenas (p) e as interações $G \times 1$ para cada grupo (Gg x E e Gp x E).

\begin{tabular}{lcc}
\hline Tratamento & QM & $\mathrm{E}(\mathrm{QM})$ \\
\hline Sementes grandes (g) & $\mathrm{QM} 1$ & $\sigma_{\mathrm{e}}^{2}+\mathrm{b} \frac{\mathrm{t}}{\mathrm{t}-1} \sigma_{\mathrm{GgE}}^{2}+\mathrm{baV}_{\mathrm{Gg}}$ \\
Gg x E & QM2 & $\sigma_{\mathrm{e}}^{2}+\mathrm{b} \frac{\mathrm{t}}{\mathrm{t}-1} \sigma_{\mathrm{GgE}}^{2}$ \\
Sementes pequenas (p) & $\mathrm{QM} 3$ & $\sigma_{\mathrm{e}}^{2}+\mathrm{b} \frac{\mathrm{t}}{\mathrm{t}-1} \sigma_{\mathrm{GpE}}^{2}+\mathrm{baV}_{\mathrm{Gp}}$ \\
Gp x E & $\mathrm{QM} 4$ & $\sigma_{\mathrm{e}}^{2}+\mathrm{b} \frac{\mathrm{t}}{\mathrm{t}-1} \sigma_{\mathrm{GPE}}^{2}$ \\
Resíduo & $\mathrm{QM} 5$ & $\sigma_{\mathrm{e}}^{2}$ \\
\hline
\end{tabular}

O coeficiente de determinação genotípica realizado nas médias de parcelas recebeu a simbologia $R_{p}^{2}$ e, nas médias de tratamentos recebeu $R_{t}^{2}$, de acordo com as fórmulas, cujos estimadores são:

$$
\mathrm{R}_{\mathrm{p}}^{2}=\frac{\hat{\mathrm{V}}_{\mathrm{G}}}{\hat{\mathrm{V}}_{\mathrm{G}}+\hat{\mathrm{\sigma}}_{\mathrm{GE}}^{2}+\hat{\mathrm{\sigma}}_{\mathrm{e}}^{2}} \text { nas parcelas }
$$

$$
\mathrm{R}_{\mathrm{t}}^{2}=\frac{\hat{\mathrm{V}}_{\mathrm{G}}}{\hat{\mathrm{V}}_{\mathrm{G}}+\frac{\mathrm{t}}{\mathrm{t}-1}\left(\frac{1}{\mathrm{a}}\right) \hat{\sigma}_{\mathrm{GE}}^{2}+\frac{\hat{\sigma}_{\mathrm{e}}^{2}}{\mathrm{ba}}} \text { em médias de tratamentos }
$$

sendo:

$\hat{\mathrm{V}}_{\mathrm{G}}$ : estimador de diversidade genética interpopulacional (entre topocruzamentos);

$\hat{\sigma}_{\mathrm{GE}}^{2}$ : estimador da variância da interação G x E;

$\hat{\sigma}_{\mathrm{e}}^{2}$ : estimador da variância do erro médio;

t: número de tratamentos;

a: número de épocas;

b: número de blocos por época.

\section{RESULTADOS E DISCUSSÃO}

As estimativas do coeficiente de determinação genotípica (Tabela 3) apresentam valores relativos às médias de topocruzamentos, entre $57,57 \%$ a 95,55\% nos caracteres avaliados, sendo superiores aos obtidos quanto à média de parcelas (valores en-

TABELA 2. Obtenção das estimativas das diversidades genéticas, relativas ao grupo dos topocruzamentos de sementes grandes $\left(\hat{\mathrm{V}}_{\mathrm{Gg}}\right)$, ao grupo de sementes pequenas $\left(\hat{\mathrm{V}}_{\mathrm{Gp}}\right)$, à interação de $\mathbf{G} \mathbf{x} \mathbf{E}$ no grupo dos topocruzamentos de sementes grandes $\left(\hat{\sigma}_{\mathrm{GgE}}^{2}\right)$, e ao grupo dos topocruzamentos de sementes pequenas $\left(\hat{\sigma}_{\mathrm{GpE}}^{2}\right)$.

\begin{tabular}{cc}
\hline Diversidade genética & Estimativa \\
\hline$\hat{\mathrm{V}}_{\mathrm{Gg}}$ & $(\mathrm{QM} 1-\mathrm{QM} 2) / \mathrm{ba}$ \\
$\hat{\mathrm{V}}_{\mathrm{Gp}}$ & $(\mathrm{QM} 3-\mathrm{QM} 4) / \mathrm{ba}$ \\
$\hat{\sigma}_{\mathrm{GgE}}^{2}$ & $(\mathrm{t}-1)(\mathrm{QM} 2-\mathrm{QM} 5) / \mathrm{bt}$ \\
$\hat{\sigma}_{\mathrm{GpE}}^{2}$ & $(\mathrm{t}-1)(\mathrm{QM} 4-\mathrm{QM} 5) / \mathrm{bt}$ \\
\hline
\end{tabular}

Pesq. agropec. bras., Brasília, v.35, n.11, p.2223-2228, nov. 2000 
tre $22,18 \%$ e $80,13 \%$ ), o que concorda com o citado por Bravo et al. (1980), sobre a precisão maior das médias de tratamentos em relação a média de repetições, pois a discussão dos resultados será baseada nas estimativas de médias de topocruzamentos.

Os maiores valores observados quanto ao coeficiente de determinação genotípica (Tabela 3) foram: 95,55\% para PCS; 93,92\% para APM; 91,37\% para VA; $91,18 \%$ para LVV e $90,51 \%$ para NDM, indicativos de que estes caracteres são os menos influenciados pelo ambiente, podendo ser selecionados em gerações mais precoces.

Os resultados referentes a PCS e APM (Tabela 3) concordam com o obtido por Jangale et al. (1994), em que, apesar da variação considerável, todos os genótipos testados apresentaram alta herdabilidade quanto a estes caracteres, sendo que o caráter APM foi o mais promissor para fins de seleção. $O$ valor de 90,51\% obtido para NDM foi menor que o observado no trabalho de Mayor \& Soto (1988); apesar disso, pode-se considerar que esta estimativa obtida é alta, e portanto, o caráter é altamente herdável para o grupo de genótipos avaliados.

Com relação ao PCS (Tabela 3), o valor estimado de coeficiente de determinação genotípica foi semelhante ao obtido por Mayor \& Soto (1988) e Konieczny et al. (1994), e maior em comparação ao apresentado por Rao \& Sharma (1985), Mauro et al. (1995) e Konovsky et al. (1996).

TABELA 3. Estimativas de coeficiente de determinação genotípica obtidas com base nas médias dos topocruzamentos $\left(R_{t}^{2}\right)$ e nas observações de cada parcela $\left(R_{p}^{2}\right)$, relativas a sete caracteres.

\begin{tabular}{lcc}
\hline Caráter $^{1}$ & $\mathrm{R}_{\mathrm{t}}^{2}(\%)$ & $\mathrm{R}_{\mathrm{p}}^{2}(\%)$ \\
\hline NDM & 90,51 & 63,40 \\
APM & 93,92 & 74,11 \\
AC & 79,52 & 41,98 \\
VA & 91,37 & 63,83 \\
PCS & 95,55 & 80,13 \\
PG & 57,57 & 22,18 \\
LVV & 91,18 & 61,67 \\
\hline
\end{tabular}

1 NDM: número de dias para a maturidade; APM: altura da planta na maturidade; AC: nota de acamamento; VA: nota de valor agronômico; PCS: peso de cem sementes; PG: produtividade de grãos de cada planta individual; LVV: nota de largura visual das vagens.
O coeficiente de determinação genotípica de $57,57 \%$ para PG (Tabela 3) foi menor em relação ao estimado por Mauro et al. (1995), próximo ao apresentado por Mayor \& Soto (1988) e superior ao obtido por Konieczny et al. (1994) e Konovsky et al. (1996). Os valores diferentes para PG podem ser explicados pelos genótipos empregados, sendo distintos em cada trabalho; além disso, o caráter sofre influência ambiental, causando as alterações observadas no coeficiente de determinação genotípica. $O$ tipo de parcela, em relação às dimensões, espaçamento entre plantas, entre outras variáveis, pode também ter sido a causa das variações observadas entre os trabalhos, influência importante segundo Moro et al. (1992).

O coeficiente de determinação genotípica de 93,92\% para APM (Tabela 3) foi próximo ao obtido por Konieczny et al. (1994), Mauro et al. (1995) e Konovsky et al. (1996), indicando uma maior herdabilidade em relação a este caráter, sofrendo menor influência ambiental. Para AC foi obtido o valor de $79,52 \%$, que se pode considerar alto, porém não foram encontrados resultados para este caráter na literatura.

Na Tabela 4 são apresentadas as estimativas das diversidades genéticas referentes ao grupo de topocruzamentos envolvendo parentais de soja tipo alimento de sementes grandes $\left(\hat{\mathrm{V}}_{\mathrm{Gg}}\right)$, para o grupo envolvendo sementes pequenas $\left(\hat{\mathrm{V}}_{\mathrm{Gp}}\right)$, suas respectivas estimativas da variância da interação $\mathrm{G} \times \mathrm{E}$ $\left(\hat{\sigma}_{\mathrm{GgE}}^{2}, \hat{\sigma}_{\mathrm{GpE}}^{2}\right)$ e os quocientes $\hat{\mathrm{V}}_{\mathrm{Gg}} / \hat{\mathrm{V}}_{\mathrm{Gp}}$ e $\hat{\sigma}_{\mathrm{GgE}}^{2} / \hat{\sigma}_{\mathrm{GpE}}^{2}$; esses quocientes representam a relação de superioridade ou inferioridade de diversidade genética disponível para seleção de um grupo em relação ao outro.

Os dados da Tabela 4 mostram que a diversidade genética dos topocruzamentos de sementes grandes foi menor que a diversidade genética de sementes pequenas em relação aos caracteres NDM, APM e $\mathrm{AC}$, pois a relação $\hat{\mathrm{V}}_{\mathrm{Gg}} / \hat{\mathrm{V}}_{\mathrm{Gp}}$ mostra que os topocruzamentos de sementes grandes possuem apenas $71 \%$ da diversidade genética apresentada pelos topocruzamentos de sementes pequenas para NDM; $87 \%$ para APM e $56 \%$ para AC. Pela relação $\hat{\mathrm{V}}_{\mathrm{Gg}} / \hat{\mathrm{V}}_{\mathrm{Gp}}$ referente aos caracteres VA, PCS, PG e LVV os topocruzamentos com sementes grandes apresentaram diversidade genética 300\%, 983\%, 45\% e 
$650 \%$, respectivamente, a mais que os de sementes pequenas, ou seja, existe maior variabilidade disponível para os topocruzamentos de sementes grandes nestes caracteres. Para as estimativas das interações G x E, somente os caracteres APM e AC apresentaram relações significativas. Observando a relação $\hat{\sigma}_{\mathrm{GgE}}^{2} / \hat{\sigma}_{\mathrm{GpE}}^{2}$, tem-se que $\hat{\sigma}_{\mathrm{GgE}}^{2}$ foi $20 \%$ maior que a $\hat{\sigma}_{\mathrm{GpE}}^{2}$ para APM, ou seja, houve maior interação do grupo dos topocruzamentos de sementes grandes com os ambientes. Já no caráter AC ocorreu menor valor para $\hat{\sigma}_{\mathrm{GgE}}^{2}$, representando apenas $10 \%$ do observado para a $\hat{\sigma}_{\mathrm{GpE}}^{2}$, e neste caso, o grupo dos topocruzamentos de sementes pequenas interagiram mais com os ambientes.

Quanto às estimativas de diversidade genética (Tabela 4), os caracteres NDM, APM e AC apresentaram valores maiores no grupo dos topocruzamentos com sementes pequenas, o que indica maior possibilidade de seleção em relação ao de sementes grandes. Nos caracteres VA, PCS, PG e LVV, as estimativas foram maiores no grupo dos topocruzamentos com sementes grandes, permitindo maiores possibilidades de seleção em relação ao outro grupo.

Deve-se observar que em relação aos caracteres NDM, APM, AC e PG, a relação $\hat{\mathrm{V}}_{\mathrm{Gg}} / \hat{\mathrm{V}}_{\mathrm{Gp}}$ apresentou valores de $71 \%, 87 \%, 56 \%$ e $45 \%$, respectivamente, indicativos de que no processo de seleção haverá diversidade genética para seleção tanto no grupo dos topocruzamentos com sementes grandes como no de sementes pequenas (Tabela 4). Já para os caracteres VA, PCS e LVV, com valores $300 \%, 983 \%$ e $650 \%$, respectivamente, maiores para grupo dos topocruzamentos de sementes grandes, a disponibilidade para seleção de materiais neste grupo é bem superior em relação a sementes pequenas.

$\mathrm{Na}$ estimativa da variância da interação $\mathrm{G} \times \mathrm{E}$, representada por $\hat{\sigma}_{\mathrm{GgE}}^{2} \mathrm{e} \hat{\sigma}_{\mathrm{GpE}}^{2}$ (Tabela 4), são observados valores altos para NDM, APM e PG no grupo dos topocruzamentos de sementes grandes, e no grupo dos topocruzamentos de sementes pequenas, para o caráter APM. Porém, é importante relacionar os valores de $\hat{\sigma}_{\mathrm{GgE}}^{2}$ e $\hat{\sigma}_{\mathrm{GpE}}^{2} \operatorname{com} \hat{\mathrm{V}}_{\mathrm{Gg}}$ e $\hat{\mathrm{V}}_{\mathrm{Gp}}$, para quantificar comparativamente a proporção entre estes estimadores; com isso, as proporções mais problemáticas são observadas para $\hat{\sigma}_{\mathrm{GgE}}^{2}(103,25)$, bem superior a $\hat{\mathrm{V}}_{\mathrm{Gg}}$ para o caráter $\mathrm{PG}$, e para o caráter $\mathrm{AC}$ tem-se que os valores estimados para $\hat{\sigma}_{\mathrm{GgE}}^{2}$ e $\hat{\sigma}_{\mathrm{GpE}}^{2}$ também foram superiores em relação às suas respectivas $\hat{\mathrm{V}}_{\mathrm{Gg}}$ e $\hat{\mathrm{V}}_{\mathrm{Gp}}$, e com isso, existe maior efeito ambiental para estes caracteres, o que é comprovado

TABELA 4. Estimativas de diversidade genética de sete caracteres do grupo dos topocruzamentos de sementes grandes $\left(\hat{V}_{\mathrm{Gg}}\right)$; do grupo dos topocruzamentos de sementes pequenas ( $\left.\hat{\mathrm{V}}_{\mathrm{Gp}}\right)$; da interação G x E do grupo de topocruzamentos de sementes grandes $\left(\hat{\sigma}_{\mathrm{GgE}}^{2}\right)$; da interação $\mathbf{G} \mathbf{x} \mathbf{E}$ do grupo de topocruzamentos de sementes pequenas $\left(\hat{\sigma}_{\mathrm{GpE}}^{2}\right)$; representação da superioridade ou inferioridade do grupo dos topocruzamentos de sementes grandes em relação ao grupo de sementes pequenas $\left(\hat{\mathrm{V}}_{\mathrm{Gg}} / \hat{\mathrm{V}}_{\mathrm{Gp}}\right)$; representação da superioridade ou inferioridade dos efeitos da interação $\mathbf{G} \mathbf{x} \mathbf{E}$ do grupo dos topocruzamentos de sementes grandes em relação ao grupo de sementes pequenas $\left(\hat{\sigma}_{\mathrm{GgE}}^{2} / \hat{\sigma}_{\mathrm{GpE}}^{2}\right)$.

\begin{tabular}{lrrrccc}
\hline Caráter $^{1}$ & \multicolumn{1}{c}{$\hat{\mathrm{V}}_{\mathrm{Gg}}$} & \multicolumn{1}{c}{$\hat{\mathrm{V}}_{\mathrm{Gp}}$} & $\hat{\mathrm{V}}_{\mathrm{Gg}} / \hat{\mathrm{V}}_{\mathrm{Gp}}$ & $\hat{\sigma}_{\mathrm{GgE}}^{2}$ & $\hat{\sigma}_{\mathrm{GpE}}^{2}$ & $\hat{\sigma}_{\mathrm{GgE}}^{2} / \hat{\sigma}_{\mathrm{GpE}}^{2}$ \\
\hline NDM & 53,00 & 75,13 & 0,71 & 4,19 & - & - \\
APM & 257,93 & 298,12 & 0,87 & 9,07 & 7,59 & 0,20 \\
AC & 0,09 & 0,16 & 0,56 & 0,02 & 0,21 & - \\
VA & 0,16 & 0,04 & 4,00 & - & - & - \\
PCS & 8,45 & 0,78 & 10,83 & - & - & - \\
PG & 75,47 & 51,92 & 1,45 & 103,25 & 0,02 & - \\
LVV & 0,15 & 0,02 & 7,50 & 25 & - \\
\hline
\end{tabular}

1 NDM: número de dias para a maturidade; APM: altura da planta na maturidade; AC: nota de acamamento; VA: nota de valor agronômico; PCS: peso de cem sementes; PG: produtividade de grãos de cada planta individual; LVV: nota de largura visual das vagens.

2 Valores não calculados. 
pelos menores coeficientes de determinação genotípica $(79,52 \%$ para AC e $57,57 \%$ para $\mathrm{PG}$ ) entre todos os caracteres avaliados.

\section{CONCLUSÕES}

1. Os topocruzamentos com materiais exóticos de soja tipo alimento apresentam coeficiente de determinação genotípica semelhante aos existentes na literatura.

2. O efeito ambiental é mais intenso nos caracteres acamamento e produtividade de grãos do grupo dos topocruzamentos de sementes grandes; e no acamamento do grupo dos topocruzamentos de sementes pequenas.

3. A variabilidade disponível para seleção no grupo dos topocruzamentos de sementes grandes é maior quanto ao valor agronômico, ao peso de cem sementes, à produtividade de grãos e à largura visual das vagens; e no grupo dos topocruzamentos de sementes pequenas, é maior quanto ao número de dias para maturidade, à altura da planta na maturidade, e ao acamamento.

\section{AGRADECIMENTOS}

Ao CNPq, pelo auxílio financeiro concedido; à Vera Maria Quecini pela correção do abstract.

\section{REFERÊNCIAS}

BRAVO, J.A.; FEHR, W.R.; CIANZIO, S.R. de. Use of pod width for indirect selection of seed weight in soybeans. Crop Science, Madison, v.20, p.507-510, 1980.

CARRÃO-PANIZZI, M.C. Valor nutritivo da soja e potencial de utilização na dieta brasileira. Londrina : Embrapa-CNPSo, 1988. 13p. (Embrapa-CNPSo. Documentos, 29).

FEHR, W.R.; CAVINESS, C.E. Stages of soybean development. Ames : Iowa State University of Science and Technology, 1977. 12p. (Special Report, 80).
JANGALE, C.B.; BIRARI, S.P.; APTE, U.B. Genetic variability and heritability in soybean. Agricultural Science Digest, Karnal, v.14, n.2, p.117-120, 1994.

JOHNSON, H.W.; ROBINSON, H.F.; COMSTOCK, R.E. Estimates of genetic and environmental variability in soybeans. Agronomy Journal, Madison, v.47, n.2, p.477-483, 1955.

KONIECZNY, G.; MUSZYNSKI, A.; NAWRACALA, J. Heritability, discrimination power, year to year variation of some characteristics and main component configuration of soybean genotypes. Soybean Genetics Newsletter, Ames, v.21, p.149-152, 1994.

KONOVSKY, J.; EVANS, D.W.; LUMPKIN, T.A. Heritability of yield, plant architecture, and quality traits of Edamame: the vegetable soybean. Soybean Genetics Newsletter, Ames, v.23, p.243-249, 1996.

MAURO, A.O.; SEDIYAMA, T.; SEDIYAMA, C.S. Estimativas de parâmetros genéticos em diferentes tipos de parcelas experimentais em soja. Pesquisa Agropecuária Brasileira, Brasília, v.30, n.5, p.667-672, maio 1995.

MAYOR, Z.F.; SOTO, J.A. Varianza genética y heredabilidades en sentido amplio en soya. Ciencias de la Agricultura, Havana, v.33, p.66-77, 1988.

MORO, G.L.; REIS, M.S.; SEDIYAMA, C.S.; SEDIYAMA, T.; OLIVEIRA, A.B. Correlações entre alguns caracteres agronômicos em soja (Glycine $\max ($ L.) Merrill). Revista Ceres, Viçosa, v.39, n.223, p.225-232, 1992.

RAO, S.K.; SHARMA, S.M. Genetic variation for pod characteristics and their inter-relationships with field germinability in soybean. Indian Journal of Agricultural Research, Karnal, v.19, n.3, p.150-154, 1985.

VELLO, N.A. Ampliação da base genética do germoplasma e melhoramento da soja na ESALQUSP. In: SIMPÓSIO SOBRE CULTURA E PRODUTIVIDADE DA SOJA, 1., 1991, Piracicaba. Anais. Piracicaba : FEALQ, 1992. p.60-81.

VENCOVSKY, R. Componentes da variação fenotípica: análise em vários ambientes. In: VENCOVSKY, R.; BARRIGA, P. (Ed.). Genética biométrica no fitomelhoramento. Ribeirão Preto : Sociedade Brasileira de Genética, 1992. p.233-333. 